Sustainable Development Goals

\section{Mehr Mut zur politischen Gestaltung}

\author{
Die Millennium-Entwicklungsziele der Vereinten Nationen werden \\ aller Voraussicht nach nicht erreicht. Nach deren Auslaufen \\ im nächsten Jahr sollen globale Nachhaltigkeitsziele die Entwick- \\ lungsrichtung vorgeben. Die Verhandlungen zu der neuen \\ Entwicklungsagenda sind in vollem Gange. \\ Von Günther Bachmann und Dorothee Braun
}

M it dem Jahr 2016 soll nach Beschluss der Vereinten Nationen eine neue Entwicklungsagenda, die Post2015-Agenda beginnen. Diese Agenda soll, so die Vereinten Nationen, eine völlig neue Qualität haben. Ein erster Vorschlag für ein neues, globales Zielsystem liegt nun vor.

Die globalen Nachhaltigkeitsziele, Sustainable Development Goals (SDG), sollen universell gültig, ihre Ziele messbar, ihre Fortschritte überprüfbar und öffentlich zugänglich sein. Sie sollen außerdem mit den fortzuschreibenden Millennium-Entwicklungszielen verbunden werden.

\section{Neues globales Zielsystem}

Mit dem neuen Zielsystem soll der drastischen Veränderung der geopolitischen Situation und der Verflechtung von sozio-ökonomischen, technologischen und ökologischen Krisenprozessen begegnet werden. Die sogenannte universelle Gültigkeit bedeutet, dass diese Ziele von allen Nationen zu verfolgen sind. Diese globale und universelle Gültigkeit wurde zumindest als politischer Anspruch formuliert.

Wie dieser konkret ausgestaltet werden wird, ist allerdings noch nicht erkennbar. Bei dem gegenwärtigen Stand der Verhandlungen ist aber zumindest eines zu erkennen: Die Post-2015-Agenda fordert von allen Beteiligten - von den politischen Akteuren und eben auch vom privaten Sektor - die volle Aufmerksamkeit ein.

\section{Neues Verfahren zur Erarbeitung}

Die Idee, globale Nachhaltigkeitsziele zu entwickeln, trägt die Handschrift der Länder Guatemala und Kolumbien. Sie fordern transformative Veränderungen auch in den Industrieländern und, dass die Armutsbekämpfung und der unbestrittene Anspruch auf wirtschaftliche Entwicklung nicht länger unabhängig von der Frage ihrer Nachhaltigkeit, von den Folgen für Natur und Gesellschaft anzugehen sein darf.

Das Neue liegt nicht nur in der universellen Adressierung der gemeinsamen, aber differenzierten Verantwortung, sondern auch im Verfahren zur Erarbeitung der SDG. Erstmals haben die Mitgliedsstaaten der Vereinten Nationen eine zwischenstaatliche offene Arbeitsgruppe eingerichtet. Erstmals wurde nicht gleich im hartnäckigen Positionskampf um Formulierungen gerungen, sondern zunächst einmal eine ausgedehnte Aufklärungsphase vereinbart und mögliche Zielvorstellungen sondiert. Deren Ergebnis hat man mit Spannung erwartet. Jetzt liegt der Entwurf eines Zielkatalogs vor.

$\mathrm{Ab}$ Herbst sollen darüber die Diplomaten verhandeln. Inhaltlich wird das Ergebnis allerdings nur stellenweise dem Leitbild einer nachhaltigen Entwicklung gerecht. Nur ansatzweise gibt es den roten Faden, entlang dessen Teilpolitiken besser verzahnt und deren Integration und Langzeitperspektive begründet werden würde.

\section{Exemplarische Entwicklung eines Ziels}

Wie ein einzelnes der SDG idealerweise aussehen könnte, das die gemeinsame Verantwortung des Nordens und des Süden bei Wahrung der unterschiedlichen Möglichkeiten sinnvoll und mit neuen Akzenten verknüpft, hat der Nachhaltigkeitsrat exemplarisch am Beispiel der Ressourceneffizienz formuliert.

Das SDG Ressourceneffizienz ist durch eine begrenzte Zahl von Handlungsfeldern und mit quantifizierten Teilschritten beschrieben. Sie können auf nationaler Ebene angepasst und umgesetzt werden. Darin sind die Vermeidung nicht wiederverwertbaren Abfalls, die Reduzierung der Bodendegradation wie der Lebensmittelverschwendung und die Stärkung der Rohstoffverantwortung entlang der Wertschöpfungskette ausgewiesen. Der Rat hat dieses Beispiel gewählt, um auf die Handlungsverpflichtung Deutschlands hinzuweisen, die aufgrund seines spezifisch großen Ressourcenkonsums und seiner Exportstärke besonders groß ist.

Weltweit ist der Bedarf an jeweils eigenständigen, systematischen Lösungen ebenso unbestritten wie die Notwendigkeit, diese an globalen Stoffströmen auszurichten. Die nationale Implementation eines universellen Nachhaltigkeitszieles zur Ressourceneffizienz bietet gute Chancen, national technologische Kompetenzen und gesellschaftliche Veränderungen voranzutreiben sowie Ingenieurskapazitäten in Entwicklungsländern aufzubauen und zur Bekämpfung von Armut und ungesunden Arbeitsbedingungen beizutragen.

\section{Transformation der globalen Ökonomie}

Eine Transformation der globalen Ökonomie, eine Erweiterung von Lebensperspektiven durch Arbeit und Minderung von Armut erfordert die Beiträge von Unternehmen. Ihr Engagement zur Nachhaltigkeit zeigt sich in der Art ihrer Unternehmensführung und in den Investitionen in eine nachhaltige Entwick- 
lung. Das schließt die Wahrnehmung der Sorgfaltspflicht im Hinblick auf Menschenrechte, Umweltrisiken und Verteilungsfragen, die Umstellung von Produkten, Prozessen und Dienstleistungen, das Angebot an ressourceneffizienten Technologien und Systemlösungen zur Nachhaltigkeit, Transparenz und Rechenschaftslegung ein.

Doch reicht es nicht, wenn sich der Privatsektor allein als Anbieter von Lösungen versteht. Lösungen zur Nachhaltigkeit entstehen dort, wo gesellschaftliche Regeln neue Märkte schaffen und alte verdrängen. Die Komplexität der Herausforderungen und Verantwortung erfordert einen kontinuierlichen und vertieften Dialog zwischen Politik und Wirtschaft.

Für die nachhaltige Entwicklung der sogenannten Entwicklungsländer klafft eine Finanzierungslücke auf Basis heutiger Investitionen von jährlich 2,5 Billionen US-Dollar (UNCTAD 2014). Wer sie schließen will, muss neue Wege gehen. Neue Wege sind notwendig in den Geschäftsmodellen im Hinblick auf Armutsminderung und Diversifizierung der Wirtschaft, im Aufbau von Partnerschaften und ihrer Rechenschaftslegung, in den Instrumenten zur Risikoabsicherung gerade kleiner und mittlerer Unternehmen und in der Kreditvergabe, hinsichtlich der Rolle und Verantwortung des öffentlichen und privaten Sektors.

\section{Umsetzung auf nationaler Ebene}

Sehr wahrscheinlich wird der nationalen Implementation der Post-2015-Agenda eine wichtige Rolle zukommen. Alles andere wäre kaum effektiv. Die Frage ist also, ob die nationale Ebene hierauf hinreichend vorbereitet ist. Zweifel sind angebracht.

Wo die große Transformation groß werden soll, muss sie im Kleinen anfangen. Das weiß man in vielen Staaten der Welt und man weiß auch, dass es regelmäßig an Kompetenzen, Kapazitäten und Kapital fehlt, um nationale Nachhaltigkeitsstrategien aufzustellen. Sie wären nämlich im Prinzip der geeig- nete Rahmen, um Querschnittsthemen zu integrieren, schrittweises Vorgehen in einer längeren Perspektive zu vereinbaren und Plattformen zur Beteiligung interessierter und betroffener Kreise zu schaffen.

\section{Ausblick}

Deutschland hat eine solche Strategie seit 13 Jahren. Das ist zwar gut, aber nicht beruhigend. Denn auch hier sind viele Dinge zu überdenken und zu entwickeln: Wie kann die nationale Nachhaltigkeitsstrategie mit ihren Zielen und Indikatoren mit der weltweiten Nachhaltigkeitsagenda verknüpft werden? Wie schafft man es, Nachhaltigkeitsregeln in das Wirtschaften zu integrieren und den Schritt von der Nische in den breiten Markt zu machen? Gute Vorschläge aus der Praxis und aus der wissenschaftlichen Begleitung würden helfen, die Leistungen zur Zielerreichung $\mathrm{zu}$ fördern und zu fordern.

\section{Literatur}

United Nations Conference on Trade and Development (UNCTAD) (2014): World Investment Report. Im Internet unter: http://unctad.org/en/ PublicationsLibrary/wir2014_en.pdf

\section{AUTOREN + KONTAKT}

Dr. Günther Bachmann ist Generalsekretär des Rates für Nachhaltige Entwicklung und Honorarprofessor der Universität Lüneburg.

Dorothee Braun ist Referentin in der Geschäftsstelle des Rates für Nachhaltige Entwicklung.

Rat für Nachhaltige Entwicklung, Geschäftsstelle c/o GIZ, Potsdamer Platz 10, 10785 Berlin. Tel.: +49 30 338424-124, E-Mail: guenther.bachmann@nachhaltigkeitsrat.de, dorothee.braun@nachhaltigkeitsrat.de, Internet: www.nachhaltigkeitsrat.de
Worüber wir heute streiten

Über kaum etwas wird so viel diskutiert wie über den Klimawandel - leider ohne sinnvolle Ergebnisse. Für den britischen Klimaforscher Mike Hulme ist er weit mehr als ein naturwissenschaftliches Phänomen; der Klimawandel ist eine kulturelle Herausforderung, ein nkomplexes Problem in einer komplexen Weltu, weshalb technisch-politische Ansätze zu kurz greifen. Das Buch hilft zu verstehen, was uns am Handeln hindert und plädiert für eine neue Sichtweise auf das Jahrhundertproblem.

\section{Hulme}

\section{Streitfall Klimawandel}

Warum es für die größte Herausforderung keine einfachen Lösungen gibt

384 Seiten, Hardcover mit Schutzumschlag, 24,95 Euro, ISBN 978-3-86581-459-3

\section{/ll oekom}

Die guten Seiten der Zukunft

Erhältlich bei www.oekom.de oekom@verlegerdienst.de

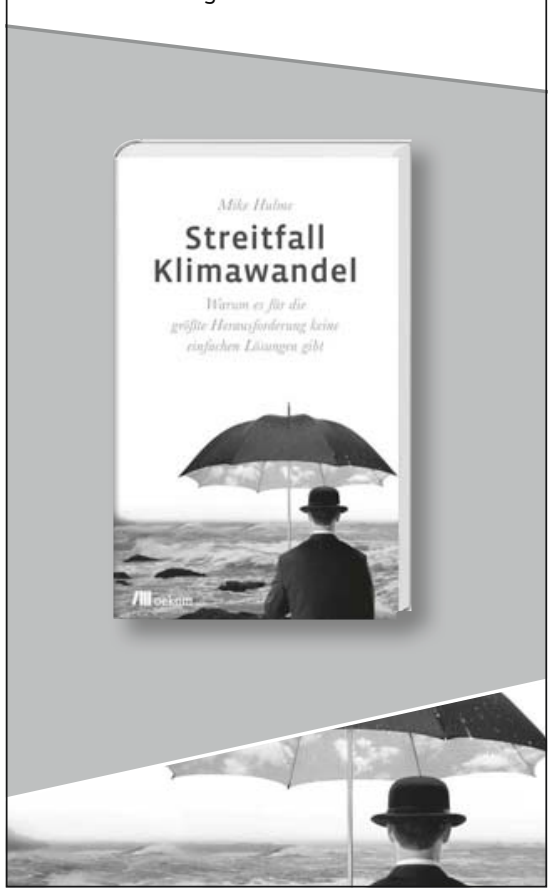

